\title{
La Psicoprofilaxis Obstétrica en gestantes adolescentes: Características y beneficios integrales
}

\author{
Maribel Yábar Peña ${ }^{1, \mathrm{a}}$
}

\section{RESUMEN}

El objetivo del presente estudio fue conocer las características y beneficios que ofrece la Psicoprofilaxis Obstétrica (PPO) en gestantes adolescentes. Asimismo, conocer en qué medida asisten al control prenatal, a las sesiones de PPO, qué tipo de parto tuvieron y su actitud respecto de la llegada del bebé y la lactancia materna entre otras variables. Se encuestó a 38 gestantes adolescentes del sector privado encontrándose que el $97 \%$ de ellas, asistieron a por lo menos 7 controles prenatales, el 100\% asistió a 6 de 8 Sesiones de PPO, 86.84\% ingresaron en fase activa de trabajo de parto. Se pudo determinar las características de las sesiones de PPO en adolescentes, las cuales mejoraron los procesos de la preparación y los resultados clínicos.

Se concluyó, que la PPO es una preparación integral que beneficia a la madre y el bebé. Debería recomendarse como parte de la atención prenatal. (Horiz Med 2014; 14(2): 39-44)

Palabras clave: Psicoprofilaxis obstétrica, gestante adolescente, beneficios. (Fuente: DeCS BIREME).

\section{Obstetric Psychoprophylaxis in teenage pregnants: Features and benefits}

\section{ABST RACT}

The aim of this study was to determine the features and benefits offered by Obstetric psychoprophylaxis (OPP) in teenage pregnants. Also, to the extent to attend prenatal care sessions PPO, what type of birth had and their attitude to the arrival of the baby and breastfeeding among other variables. We surveyed 38 pregnant teenagers in the private sector was found that $97 \%$ of them attended at least 7 prenatal visits, $100 \%$ attended 6 of 8 sessions of PPO, $86.84 \%$ were admitted in the active phase of labor. It was determined the characteristics of adolescents PPO sessions, which improve the processes of preparation and clinical outcomes.

It was concluded that the PPO is their a comprehensive preparation for the major benefits offered to the mother and baby should be recommended as part of your prenatal care. (Horiz Med 2014; 14(2): 39-44)

Key words: Obstetric psychoprophylaxis, teenage pregnant, benefits. (Source: DeCS BIREME)

Licenciada en Obstetricia.

a Miembro Titular de la Sociedad Peruana de Psicoprofilaxis Obstétrica. Lima, Perú. 


\section{INTRODUCCIÓN}

La gestación es una etapa especial para cada mujer, independientemente de su situación personal, médica o socio-cultural. La adaptación a los cambios físicos, emocionales y cognitivos, van a tener una respuesta diferente influenciada por las características psicológicas, las perspectivas, el entorno y la edad de la gestante.

Podría ser una vivencia positiva y feliz, aún con una serie de expectativas y temores, 0 en otras situaciones, podría tratarse de una jornada negativa y llena de conflictos, como en el caso de las adolescentes. (1)

El Perú, aún refleja una alta tasa de gestación en adolescentes, lo cual no ha variado en los últimos 20 años, ubicándose como una prioridad en la salud pública, por sus relevantes consecuencias médicas, sociales y económicas, tanto para la madre, el bebé y la sociedad. De acuerdo a la Encuesta Demográfica y de Salud Familiar (ENDES) 2012, el 13.5\% de las mujeres entre los 15 y 19 años están o han estado embarazadas.

De ellas, el $57 \%$ no cuentan con ningún grado de instrucción, el $47 \%$ cuenta con educación escolar (incompleta y completa) y solo el $5 \%$ tiene educación superior, poniendo como característica clave su bajo grado de instrucción y a la vez haciéndolas más vulnerables a no poder salir del círculo de la pobreza. $(2,3)$

Asimismo el índice de mortalidad materna entre las adolescentes podría llegar a duplicarse en relación a las cifras generales. El embarazo en adolescentes es de alto riesgo porque ellas no han terminado su maduración biológica ni psicológica, por lo que tiene más probabilidades de hacer complicaciones y terminar en cesárea. Por lo tanto son un grupo especial que se tomará como "una situación especial", que demanda una preparación prenatal integral y que requerirá una atención basada en sus necesidades específicas. Si bien la mayoría de las adolescentes pueden biológicamente dar a luz bebés sanos, corren más riesgos de presentar determinadas complicaciones si no recibieron un adecuado control prenatal y psicoprofilaxis obstétrica.
Los bebes de madres adolescentes tienen de 2 a 6 veces más probabilidades de presentar complicaciones, que los de madres de 20 años 0 más. Muchas madres adolescentes no cumplen con sus controles prenatales y suelen reflejar hábitos nocivos como el consumo de tabaco, alcohol, drogas, mala alimentación, incluso alta probabilidad de poliandria.

Estos factores incrementarían los riesgos como el bajo peso al nacer, infecciones, dependencia química, desproporción céfalo pélvica, anemia, hipertensión entre otras. (4)

Todo ello, hace necesario que el sistema de salud cuente con un servicio de atención integral adaptado a esta situación especial, que de acuerdo a la clasificación de la Psicoprofilaxis Obstétrica (PPO) en Situaciones Especiales, según ASPPO (Sociedad Peruana de Psicoprofilaxis Obstétrica), reunirá condiciones personales, médicas y socioculturales, las cuales por su naturaleza demandarán un programa de preparación integral especialmente dirigido para adolescentes, durante el embarazo parto y puerperio, en el que se pueda desarrollar el componente teórico, físico y psico-afectivo, según sus propias necesidades, para lograr una jornada obstétrica en las mejores condiciones con una experiencia saludable y positiva tanto para la madre como para el bebé, incluyendo como parte del proceso el vínculo afectivo de la familia. (5)

\section{EL ESTUDIO}

Se realizó un estudio observacional, descriptivo y transversal en 38 gestantes adolescentes de la consulta privada en la ciudad de Lima. Se aplicó una encuesta que incluyó variables como: la asistencia un mínimo de 7 controles prenatales, Asistencia a más de 6 de 8 sesiones de PPO, la hospitalización en fase activa, parto vaginal vs cesárea, uso de epidural, lactancia materna exclusiva, actitud positiva ante la llegada del bebé al inicio y al final de la PPO y la asistencia al control postnatal. Se elaboró una plantilla en Excel con los detalles respectivos, lista para ser llenada de acuerdo a los resultados observados. 


\section{HALLAZGOS}

Las gestantes adolescentes que reciben PPO mostraron mayor interés y responsabilidad respecto de asistir a su control prenatal, es así que el $97.37 \%$ de ellas recibieron más de 7 controles prenatales, el 100\% recibió al menos 6 de un total de 8 sesiones prenatales, $86.84 \%$ ingresaron en fase activa para su parto, el mismo porcentaje $86.84 \%$ tuvo parto vaginal, mientras que el $13.16 \%$ lo tuvo por cesárea,

Asimismo, el 7.89\% utilizó la epidural, el $92.11 \%$ optó por la lactancia materna exclusiva, el $39.47 \%$ tenía una actitud positiva ante la llegada del bebé al inicio de las sesiones de PPO, mientras que el esta cifra se invirtió al final de las sesiones de PPO, lográndose una actitud positiva ante la llegada del bebé en el $100 \%$ de las gestantes adolescentes.

Se observó también que de las 38 pacientes, el 100\% asistió a su control postnatal. Tabla 1. presentarse en una edad especial (de cambios), requiere de una atención diferenciada, con un enfoque que nos permita reconocer todos los aspectos asociados, tales como: si fue una gestación planificada, si hubo o no pensamiento respecto del aborto, la presencia de mayores riesgos obstétricos y perinatales asociados a conductas no saludables, desinterés en la prole, falta de control prenatal, entre otros.

Asimismo, la posibilidad de no contar con la pareja, el riesgo de no concluir los estudios o la necesidad de trabajar, y la baja autoestima, son aspectos muy asociados a la gestación en la adolescencia.

Con frecuencia, observamos una serie de emociones intensas que influyen en su reacción y expectativas respecto a la llegada de un bebé, tales como: la falta de menstruación, con o sin deseo de la maternidad, el test del embarazo positivo, la comunicación con la pareja, la primera ecografía, las primeras sensaciones

Tabla 1. Beneficios de la PPO en gestantes adolescentes.

Variables

Asistencia a más de 7 Controles prenatales

Asistencia a más 6 sesiones de PPO de un total de 8

Hospitalización en dase activa

Parto vaginal

Parto por cesárea

Uso de epidural

Lactancia materna exclusiva

Actitud positiva ante la llegada del bebé al inicio de la PPO

Actitud positiva ante la llegada del bebé al final de la PPO

Asistencia al Control postnatal

$\mathbf{N}^{\circ}$

37

37

38

33

33

5

3

35

15

38

38
SI $\%$

97.37

100

86.84

86.84

13.16

7.89

92.11

39.47

100

100
NO $\%$

$\begin{array}{rcc}1 & 2.63 & 38 \\ 0 & 100 & 38 \\ 5 & 13.16 & 38 \\ 5 & 13.16 & 38 \\ 33 & 86.84 & 38 \\ 36 & 92.11 & 38 \\ 3 & 7.89 & 38 \\ 23 & 60.53 & 38 \\ 0 & 0 & 38 \\ 0 & 0 & 38\end{array}$

\section{DISCUSIÓN}

El presente estudio es relevante porque nos permite objetivar cuán importante es, que la gestante adolescente, cuente con una preparación integral del embarazo.

La PPO, como parte de la atención prenatal, logra despejar una serie de interrogantes, inquietudes y temores, respecto de esta nueva etapa, que por de los movimientos fetales, las primeras contracciones uterinas, el internamiento antes del nacimiento, el primer contacto físico con el bebé, la primera mirada mamá y bebé y el primer contacto del bebé con el pecho (6).

Sin embargo, a través de la PPO, que le brinda una preparación integral al incluir: información, 
entrenamiento físico y soporte psico-afectivo, la gestante adolescente puede lograr motivación, tranquilidad y expectativas positivas, lo cual se traduce en una palabra clave que es la seguridad, y ésta a su vez, significa la posibilidad de aceptar y llevar la experiencia de la maternidad de manera natural y positiva, incentivándola a participar activamente en todo el proceso, desarrollando su autoestima, motivando su autocuidado y la identificación oportuna de signos de alarma, prevenir riesgos y complicaciones a través de hábitos y etilo de vida saludable, consiguiendo su adaptación a los cambios, y disminuyendo la ansiedad y temor respecto del parto.

Otro aspecto relevante, es el impacto positivo que tiene la PPO sobre la motivación y vínculo afectivo prenatal, observándose que del $60.53 \%$ de las gestantes que no iniciaron su preparación con una actitud positiva respecto de la llegada de su bebé, al final de la preparación, se logró que el 100\% manifestara satisfacción y una actitud positiva sobre ello, lo cual fortaleció el vínculo familiar.

Si bien la PPO considera en sus contenidos, información, entrenamiento físico y preparación psico-afectiva, las sesiones con gestantes adolescentes, para que tengan mejor aceptación y resultados, debe caracterizarse a través de talleres participativos, con mucha actividad dinámica para motivar la asistencia y permanencia en el programa, sin modificar los objetivos y la labor asistencial obstétrica de la PPO.

Asimismo, es importante contestar a todas sus interrogantes, utilizando un lenguaje muy coloquial, acorde con su argot característico e incorporando ejemplo o uso de analogías sencillas y didácticas para facilitar su comprensión y motivación. (7)

El número de sesiones será de acuerdo a la evolución y necesidades de las participantes, y de preferencia debería ser exclusivamente con gestantes de su misma condición adolescente, siendo las sesiones individuales muy recomendables por tener la ventaja de brindar la preparación de manera más personalizada y sin distracciones, por lo tanto, apuntando al logro de los obj etivos.

Asimismo, al fomentar la participación de la pareja e incluso el acompañamiento en el parto, se logra estrechar más el vínculo afectivo prenatal, lo cual facilita el proceso de motivación, aceptación, acogida y afecto hacia el bebé, incluso en los casos de negación o rechazo como se observó en el $60.53 \%$ de las gestantes de la muestra, quienes no tenían una actitud positiva respecto de la crianza del bebé al inicio de su PPO, sin embargo, esta situación se revirtió en el transcurso de su preparación al punto que se logró que el $100 \%$ de las adolescentes, manifestaran acogida y afecto para con su bebé. Esto se facilita con el desarrollo de sesiones y talleres dinámicos, para trabajar la vinculación afectiva con el bebé, el padre y/ o la familia, permitiendo una mayor aceptación del embarazo. Este aspecto se facilita con apoyo de música sugestiva. Por lo tanto, el apoyo psico-afectivo se considera uno de los pilares importantes sobre los que descansa la PPO en adolescentes, y la seguridad, junto con los talleres de autoestima, aportan positivamente a este fin (8).

Es notorio que cuando la madre va recuperando su autoestima y se empodera de su salud, es consciente de lo importante que es internalizar un estilo de vida saludable, lo cual permite que valore y sea responsable de hacer su control prenatal, como lo objetivamos en el $97.37 \%$ de gestantes adolescentes que acudieron a más de 7 controles prenatales, con lo cual se redujeron sus riesgos obstétricos y perinatales, dado que con buen seguimiento se puede hacer prevención.

Esto se logra con el esfuerzo desplegado en las sesiones de PPO, informándoles y sensibilizándolas para que tengan presente lo importante de contar con su control prenatal puntual.

Asimismo, al tener más tiempo para despejar dudas y compartir sus expectativas, sienten la motivación y necesidad de acudir a sus sesiones de PPO, como lo vemos reflejado en los resultados: el $100 \%$ de las gestantes adolescentes, asistió a más de 6 de un total de 8 sesiones (9).

Para el desarrollo de las sesiones, se recomienda una ambientación adecuada de la sala de PPO, para que sea motivadora, estimulante y atractiva a las expectativas de las adolescentes gestantes, con mensajes claros, agradables, y tal vez con imágenes o personajes, y música de la preferencia y simpatía de los jóvenes. 
Todo esto la estimula, facilita su atención y hace más fácil su proceso informativo-educativo, lo cual permite que conozca aspectos sobre su gestación, parto y post parto con naturalidad, por ejemplo; aprendiendo a reconocer lo normal de lo que podría ser un signo de alarma, además de identificar bien las contracciones uterinas, lo cual mejora su actitud y desenvolvimiento durante la gestación y parto, tal como se objetivó en el $\mathbf{8 6 . 8 4 \%}$ de las madres que acudieron en fase activa para su atención del parto, dado que fueron conocedoras de las contracciones uterinas y supieron esperar hasta el momento oportuno para acudir a la clínica.

Asimismo, al tener una buena respuesta con las técnicas de autoayuda, como las técnicas de respiración, relajación, posiciones antálgicas, masajes localizados, entre otras, se correlacionó con tener un parto vaginal, como se observó en el $86.84 \%$ de las madres. Solo un $7.89 \%$ utilizó la epidural, lo cual demostraría que la PPO las ayudó a controlar mejor la sensación de molestias o dolor durante el trabajo de parto (10).

Todas las adolescentes requieren desarrollar mucho el componente afectivo y en algunos casos el trabajo multidisciplinario ayuda a que se trabaje de la mano con una psicóloga, un pediatra, un ginecólogo, entre otros profesionales, quienes refuerzan la información sobre temas específicos que favorece el conocimiento de las madres ayudándolas a tomar buenas decisiones sobre sus propios cuidados y sobre los de su bebé. Pudimos objetivar que de las 38 adolescentes, el 92.11\% eligió la lactancia materna exclusiva, por sus beneficios y motivación personal. Cabe resaltar que el 7.9\% restante, manifestó requerir la lactancia mixta por su necesidad de retomar sus estudios y ausentarse por muchas horas.

La PPO, es el espacio privilegiado para hablar sobre los cuidados del bebé y de la salud sexual y reproductiva, por ello; en la última sesión se informa a la gestante sobre puericultura y la importancia de su control anual, despistaje de cáncer ginecológico y planificación familiar, por lo que usualmente, al concluir su PPO, tienen una idea clara de la metodología anticonceptiva que elegirán para no volver a exponerse a una gestación no planificada, siendo conscientes de lo importante que es asistir a su control después del parto, lo que se reflejó en el $100 \%$ de las madres que acudieron a su control postnatal, durante el cual se suele averiguar sobre si desarrollaron el síndrome de depresión postparto, no siendo frecuente este cuadro generalmente en las madres que recibieron PPO $(11,12)$.

En conclusión, la PPO es una herramienta para mejorar las condicionesobstétricasy emocional es en la gestante adolescente, por lo que se recomienda como parte de su atención integral. Es recomendable promover en los profesionales de la Obstetricia, habilidades que le permitan establecer desde un inicio, una relación empática con las gestantes adolescentes, propiciando un clima de confianza y seguridad para el desarrollo normal del proceso del embarazo, parto y puerperio; como un acontecimiento fisiológico, natural y positivo.

Las autoridades competentes, deberían considerar el crear espacios adaptados para trabajar con este grupo de gestantes, en horarios y ambientes especiales para desarrollar la PPO con el recurso humano adecuado: con el perfil y capacitación para atenderlas con calidad, apoyándose de un ambiente acogedor, material y lenguaje apropiado, enfoque de género, derechos humanos e interculturalidad, dada la estadística y problemática a nivel nacional.

\section{Agradecimiento}

Un agradecimiento especial a todas las gestantes que colaboraron con la información para el presente estudio.

\section{Fuentes de financiamiento}

Este estudio ha sido autofinanciado por la autora.

\section{Conflictos de interés}

La autora declara no tener ningún conflicto de interés. 


\section{REFERENCIAS BIBLIOGRÁFICAS}

1. Vital M. Programa de Psicoprofilaxis Obstétrica: como debe ser en Adolescentes. Boletín Científico ASPPO 2008; 10(30): 8.

2. ENDES 2012 [Internet]. Lima, Perú: Instituto Nacional de estadística e informática; 2012 [Citado el 29 de setiembre de 2012] Disponible en: http:// proyectos. inei. gob. pe/ endes/ 2012/

3. UNFPA [Internet]. Lima, Perú: Fondo de Población de Naciones Unidas; 2013 [Citado el 15 de setiembre de 2013] Disponible en: http:/ / www. unfpa. org. pe/ eaperu

4. Evans MA, Rosen LN. Demographic and Psychosocial risk factors for preterm delivery in an active duty pregnant adolescent population. J Medicine 2000; 165: 49-53.

5. ASPPO (Sociedad Peruana de Psicoprofilaxis Obstétrica). Manual de Psicoprofilaxis Obstétrica, 3ra Ed. Lima: ASPPO. 2010.

6. Morgan DC, Chopa G, Fisher M. Psychosocial variables associated with teenager pregnancy. Adolescence 1995; 30: 277-89.
7. Morales S. La Psicoprofilaxis Obstétrica como eje de la prevención prenatal, 1a. ed. Lima: Cimagraf. 2004.

8. Gagnon AJ., Sandall J. Educación para el parto: ¿Grupal o individual?. Boletín Científico ASPPO 2011; 13(39): 2-3.

9. Toledo V, Molina R, Caris L, Luengo X, González E. Adolescente embarazada. Aspectos psico-psiquiátricos. En: Diagnóstico: Adolescentes embarazadas. CEMERA Facultad Medicina, Universidad de Chile 1991; 130-8.

10. Hospital Bertha Calderón Roque, Centro de Salud Sexual Reproductiva para Adolescentes. Manual de Procedimientos Curso Parto Psicoprofiláctico, $1^{\text {a }}$ Ed. Managua: 2010.

11. Prado I. Estrategias de afrontamiento en la calidad de vida percibida por adolescentes embarazadas. Hospital Regional Hermilio Valdizán Medrano-Huánuco 2009. Boletín Científico ASPPO 2011; 15(44): 4-5.

12. Phipps $M$, Raker $C$, Ware $C$, et al. Randomized controlled trial to prevent postpartum depression in adolescent mothers. Am J Obstet Gynecol 2013; 208:192.e1-6.

\section{Correspondencia:}

Maribel Yábar Peña

Dirección: Jr. Huamachuco 1558 Dpto. 103. Jesús María, Lima-Perú

Teléfax: +(51 1) 6503981 / 995283285

Correo electrónico: maribelyabar@hotmail.com 